\title{
A Numerical Model of Joule Heating in Piezoresistive Pressure Sensors
}

\author{
Abdelaziz Beddiaf $^{1}$, Fouad Kerrour ${ }^{2}$, Salah Kemouche ${ }^{2}$ \\ ${ }^{1}$ Faculty of Science and Technology, Khenchela University, Algeria \\ ${ }^{2}$ University of Constantine 1, MoDERNa Laboratory, Constantine, Algeria
}

\begin{tabular}{l}
\hline Article Info \\
\hline Article history: \\
Received Des 25, 2015 \\
Revised Feb 28, 2016 \\
Accepted Mar 12, 2016 \\
\hline
\end{tabular}

Keyword:

Joule Heating

Thermal Drift

Piezoresistive Pressure Sensors

Finite Difference Method

\begin{abstract}
Thermal drift caused by Joule heating in piezoresistive pressure sensors affects greatly the results in the shift of the offset voltage of the such sensors. The study of the thermal behavior of these sensors is essential to define the parameters that cause the output characteristic drift. The impact of Joule heating in a pressure sensor has been studied. The study involves the solution of heat transfer equation considering the conduction in Cartesian coordinates for the transient regime using Finite Difference Method. We determine how the temperature affects the sensor during the applying a supply voltage. For this, the temperature rise generated by Joule heating in piezoresistors has been calculated for different geometrical parameters of the sensor as well as for different operating time. It is observed that Joule heating leads to important rise temperature in the piezoresistor and, hence, causes drift in the output voltage variations in a sensor during its operated in a prolonged time. This paper put emphasis on the geometric influence parameters on these characteristics to optimize the sensor performance. The optimization of geometric parameters of sensor allows us to reducing the internal heating effect. Results showed also that low bias voltage should be applied for reducing Joule heating.
\end{abstract}

Copyright (C) 2016 Institute of Advanced Engineering and Science. All rights reserved.

\section{Corresponding Author:}

Abdelaziz Beddiaf,

Faculty of Sciences and Technology,

Khenchela University, Algeria,

Department of Electronics, University of Constantine 1, Algeria.

Email: beddiafaziz@yahoo.fr

\section{INTRODUCTION}

A silicon piezoresistive pressure sensor which use the Wheatstone-bridge have found a wide application in various domains, such as automotive engineering, aerospace and biomedical instruments. They have the advantages of high sensitivity, excellent linearity of electrical response, good technological compatibility, small size, low power, mass production and some other advantages [1]-[7]. Nevertheless, they often suffer from the temperature drift, the study of the thermal behavior of these sensors is necessary so as to determine the parameters that cause the output characteristics drift. The knowledge of the phenomenons causing its thermal drift presents a particular interest.

In his work, Yicai Sun [5] showed the existence of the electric drift of bridge offset for piezoresistive pressure sensors as well as thermal drift. His study uses the electric drift for compensating offset thermal drift. In a previous paper [8] we have investigated the effect of temperature and doping level on the characteristics of such sensors. The approach to the compensation for temperature drift of offset voltage in this sensor type was proposed by U. Aljancic [7]. The Joule heating in piezoresistive microcantilever sensors was indicated by M. Zahid [9]. His approach focuses on the analytical and numerical techniques to characterize the Joule heating in such microcantilevers. He introduced a theoretical model for predicting the temperature produced by the Joule heating. The same authors have developed a simple and 
accurate conduction-convection model to predict the temperature distribution in p-doped piezoresistive microcantilevers because of self-heating [10]. Recently, we made a thermomechanical modeling of a piezoresistive pressure sensor [11]. More recently, in another study, we modeled the thermal behavior of the capacitive pressure sensors, using Finite Element Analysis (FEA) established in COMSOL. The thermal effects due to the temperature considering the materials' properties, the geometric shape and also the heat transfer mechanisms are developed [12]. Piezoresistive pressure sensor using a Wheatstone bridge with the piezoresistors is typically used with a supply voltage ranging from 3 to $10 \mathrm{~V}$ involves thermal drift caused by Joule heating.

The present work seeks to study the impact of Joule heating in a piezoresistive pressure sensor. The study involves the solution of heat transfer equation considering the conduction in Cartesian coordinates for the transient regime using Finite Difference Method $(F D M)$. It aims to explore the geometric influence parameters on these characteristics to optimize the sensor performance. The elevation of temperature caused by the Joule heating in piezoresistors has been calculated for various geometrical parameters of the device as well as for several operating time; it allows us to optimization the sensor design for reducing the Joule heating.

\section{METHODOLOGY AND THEORY}

Joule heating, also known as ohmic heating or electrical-resistive, is the process by which the passage of an electric current through a conductor releases heat. The bulk of the thermal energy is produced due to loss of kinetic energy of current carrying electrons by collisions among themselves and with the lattice atoms [13]. In this work, we consider that the Joule heating effect as the only energy conversion, neglecting the other heat transfer modes such as the convection and radiation. The variation of temperature due to Joule heating, considering the heat transfer by conduction in the piezoresistive pressure sensors, is shown in Figure 1 .

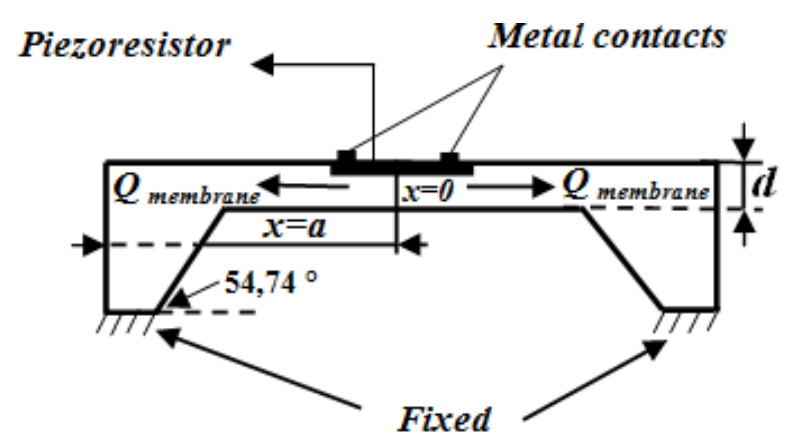

Figure 1. Piezoresistive pressure sensor structure with heat transfer path

The heat conduction equation predicting the Joule heating on the piezoresistive pressure sensor considering its three-dimensional form in Cartesian coordinates for transient regime and including thermal energy generation is given by [9]:

$$
\frac{\partial^{2} T}{\partial x^{2}}+\frac{\partial^{2} T}{\partial y^{2}}+\frac{\partial^{2} T}{\partial z^{2}}=\frac{1}{\alpha} \frac{\partial T}{\partial t}-\frac{q}{k}
$$

Where $\boldsymbol{q}$ is heat flux, $\boldsymbol{k}$ is thermal conductivity, $\boldsymbol{\alpha}$ is thermal diffusivity and $\boldsymbol{t}$ is the time. Neglecting the change in temperature along the perpendicular direction, equation (1) can be modified as:

$$
\frac{\partial^{2} T}{\partial x^{2}}+\frac{\partial^{2} T}{\partial y^{2}}=\frac{1}{\alpha} \frac{\partial T}{\partial t}-\frac{q}{k}
$$

The rate of energy generation is expressed by:

$$
q=\frac{V_{0}^{2}}{R d a^{2}}
$$


Where $\boldsymbol{V}_{\boldsymbol{0}}$ is applied electrical potential, $\boldsymbol{d}$ is the thickness of membrane, $\boldsymbol{a}$ is the length of the square-shaped membrane and $\boldsymbol{R}$ is the resistance of the diffused piezoresistor, given by the following expression [9]:

$$
R=\rho_{e} \frac{L_{p z r}}{A_{p z r}}
$$

Where $\boldsymbol{L}_{p z r}$ is the length of the piezoresistor, $\boldsymbol{A}_{p z r}$ the cross-sectional area and $\boldsymbol{\rho} \boldsymbol{e}$ is the electrical resistivity.

The applicable boundary conditions include the adiabatic heat condition and maintaining the heat continuity at the edges which are:

$$
\left\{\begin{array}{l}
\left.\frac{\partial T}{\partial x}\right|_{x=0}=0 \\
\left.\frac{\partial T}{\partial y}\right|_{y=0}=0
\end{array}\right.
$$

Taking also into account that the diffused piezoresistor can be considered a perfect conductor, we will have:

$$
\left\{\begin{array}{l}
-k \frac{\partial T}{\partial x}=\left.h\left(T-T_{0}\right)\right|_{x=a} \\
-k \frac{\partial T}{\partial y}=\left.h\left(T-T_{0}\right)\right|_{y=a}
\end{array}\right.
$$

Where $\boldsymbol{h}$ is the heat transfer coefficient that depends on the material and the surrounding air. The initial condition in the all structure is:

$$
\left.T(x, y, t)\right|_{t=0}=T_{0}
$$

\section{FINITE DIFFERENCE METHOD (FDM)}

The material properties of Silicon used in this work are indicated in Table 1.

Table 1. Material property of Silicon [9],[10]

\begin{tabular}{lc}
\hline \multicolumn{1}{c}{ Parameter } & Values \\
\hline Mass density, $\rho\left(\mathrm{kg} / \mathrm{m}^{3}\right)$ & 2320 \\
Heat transfer coefficient, $h\left(\mathrm{Wm}^{-2} \mathrm{~K}^{-1}\right)$ & 2.219 \\
Electrical resistivity, $\rho \mathrm{e}(\Omega \cdot \mathrm{m})$ & $10^{-3}$ \\
Thermal conductivity, $K\left(\mathrm{Wm}^{-1} \mathrm{~K}^{-1}\right)$ & 150 \\
Specific heat, c $(\mathrm{J} / \mathrm{KgK})$ & 712 \\
Thermal diffusivity, $\alpha\left(\mathrm{m}^{2} / \mathrm{s}\right)$ & $0.9 * 10^{-4}$ \\
\hline
\end{tabular}

\subsection{Finite Difference Method validation}

The 2D heat conduction equation in transient regime is discretized using the Finite Difference Method $(F D M)$ and the obtained system of linear equations is solved by the Thomas algorithm using the Matlab calculation software [14]. To validate the Finite Difference model, we compared the obtained results of our model with those results obtained by other authors [15].

C. Pramanik has developed an analytical model for the resolution of the heat transfer equation in cylindrical coordinates for circular structure in the transient regime using the method of superposition of variables [15].

Using their results to validate our model, we compared the results of the change in temperature as function of time with those of the analytical model (Figure 2). According to the variation of temperature as a function of time in the curve of Figure 2, we can notice that the results are in good agreement. The comparison of the obtained results allows us to validate the FDM model. 


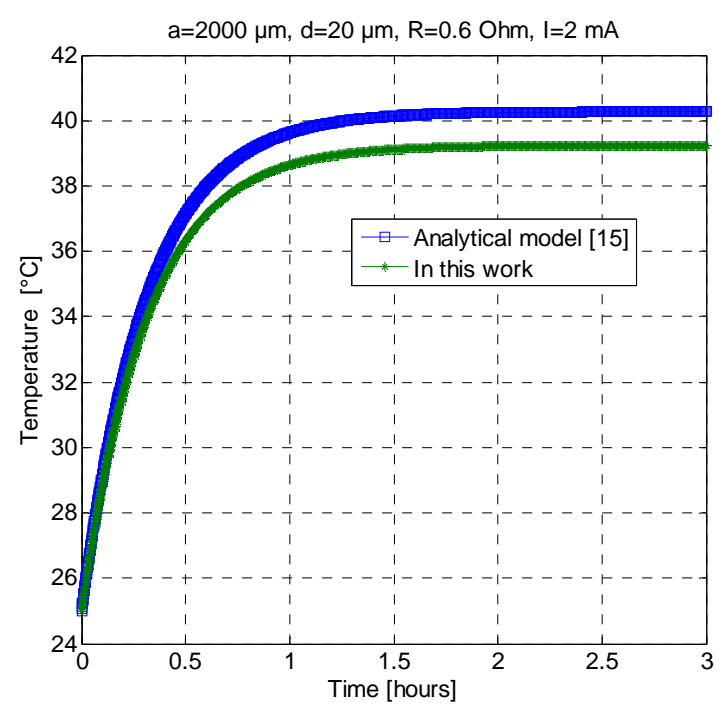

Figure 2. Variation of temperature as a function of time [15]

\subsection{Geometric effect parameters in the generation of temperature}

As previously noted, this study is devoted to the thermal behavior of the sensors. For this, we adopt the model of Finite Difference Method. The thermal drifts provoked by Joule heating in piezoresistive pressure sensors affect greatly the performance of such sensors. As we know, the geometric influence parameters on the rise of temperature have an enormous impact, we focused on the thermal drift study of the Joule heating in this sensor type. In the recent work [12], we have studied the thermal effects of capacitive pressure sensor due to the temperature taking into consideration the geometric shape, the materials' properties and also the heat transfer mechanisms.

We will study the thermal drift caused by Joule heating of piezoresistive pressure sensor characteristics, so the geometric influence parameters on these characteristics to optimize the sensor performance. The choice of these geometrical parameters for simulations is taken from earlier papers [11], [16].

\subsubsection{Effect of applied voltage in generation of temperature}

To see the effect of applied voltage on the temperature generation in the center of the piezoresistor, we varied many parameters as: the thickness of the diaphragm, the length of the membrane, geometrical piezoresistor and operating time of the device.

\subsubsection{Geometric effect parameters of the piezoresistor}

Figure 3 shows the variations in temperature as a function of applied voltage in the range of 3 to 10 $\mathrm{V}$ for several lengths of the piezoresistor $\boldsymbol{L}_{p z r}$, where the device is operating for one hour. In this case, we varied $\boldsymbol{L}_{p z r}$ with the fixing of the other geometrical parameters; we have observed that the temperature is increasing function of the applied voltage.

The $\mathrm{T}\left(\mathrm{V}_{0}\right)$ is inversely proportional to $\boldsymbol{L}_{p z r}$. So, the sensors with the low Joule heating is obtained for a great length of the piezoresistor which is a drawback. However, it can be seen in Figure 4 that the Joule heating causes a raise of the temperature by increasing the cross-sectional area $\boldsymbol{A}_{p z r}$. Noting that the temperature and applied voltage shows a parabolic dependence. 


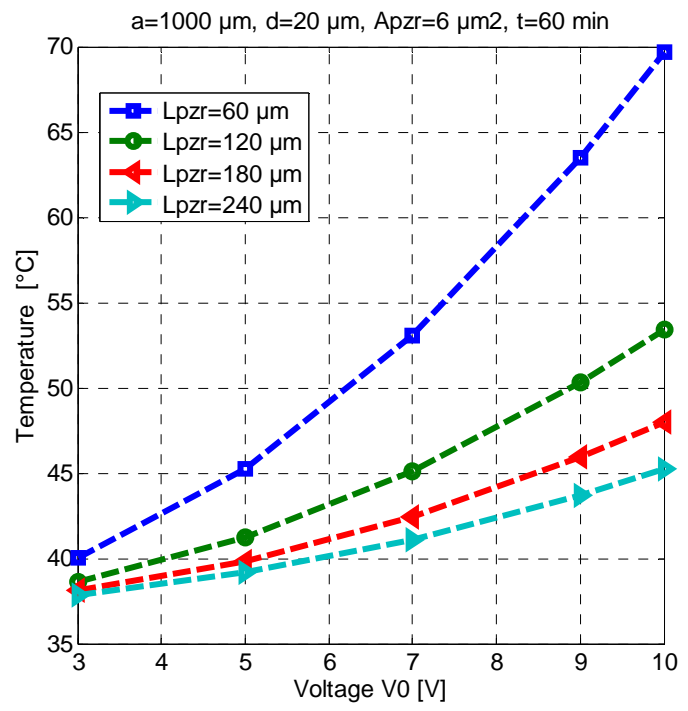

Figure 3. Variation of temperature as function of applied voltage for several lengths of the piezoresistor $\boldsymbol{L}_{p z r}$

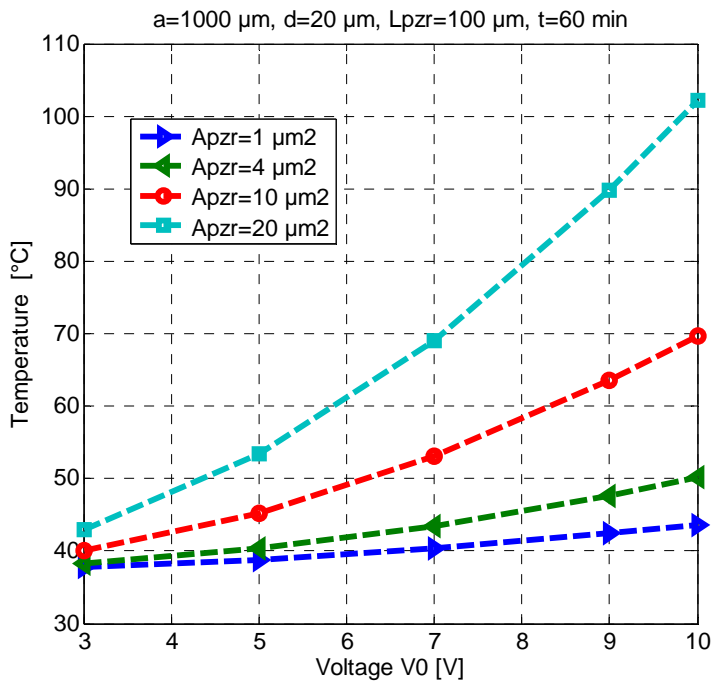

Figure 4. Variation of temperature as function of applied voltage for several cross-sectional areas $\boldsymbol{A}_{p z r}$

\subsubsection{Effect of the operating time of the sensor $t$}

Figure 5 gives the evolution of the temperature as a function of applied voltage for several values of time $t$. It is clear that the temperature is proportional to the operating time of the device. 


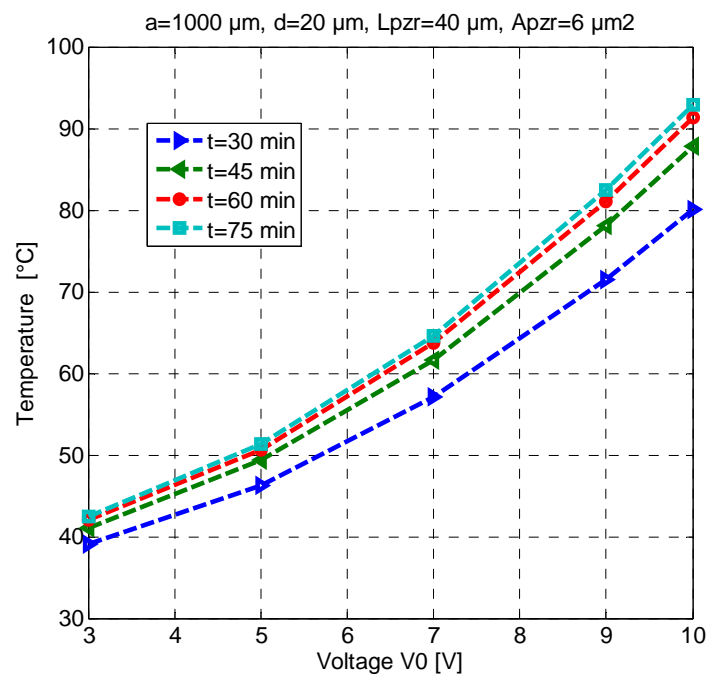

Figure 5. Temperature variation vs. applied voltage for different values of time

\subsubsection{Membrane thickness effect $d$}

To highlight the effect of the thickness of the diaphragm, we have shown in Figure 6 the temperature variation according to the applied voltage for different values of the thickness $d$. The $\mathrm{T}\left(\mathrm{V}_{0}\right)$ goes on decreasing as the membrane thickness is increased. However, according to previous studies [11],[12] this leads to weakening the pressure sensitivity of the sensors.

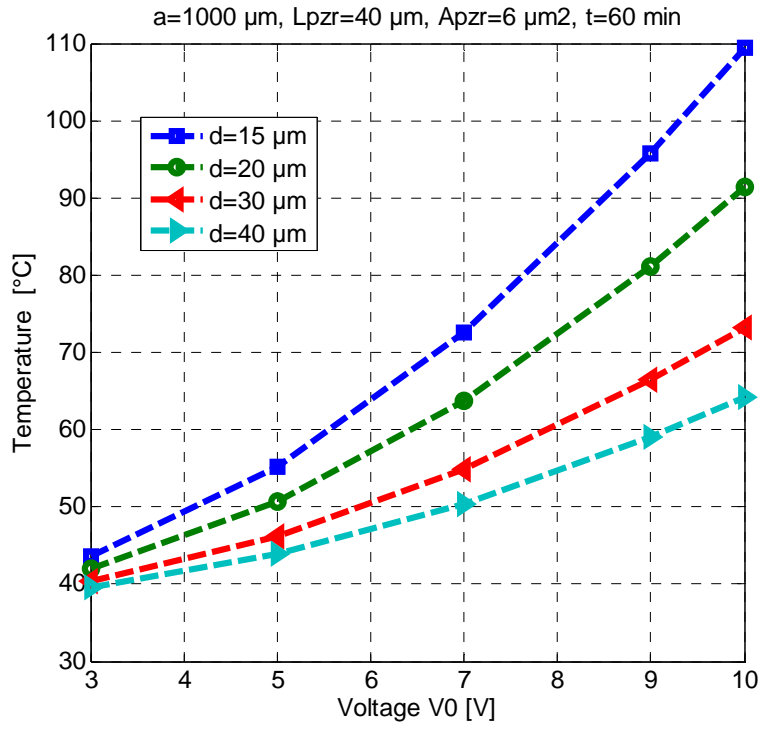

Figure 6. Temperature variation vs. applied voltage for several membrane thickness $d$

\subsubsection{Effect of side length of the membrane $a$}

Based on the results shown in Figures 7 and 8, it can be observed that the temperature rise generated by the Joule heating in piezoresistive pressure sensors is a decreasing function of the side length of the diaphragm $a$. So, to reduce this effect, it is required to have a large side length. This solution is easy to establish and does not affect the pressure sensitivity. Nevertheless, it leads to the enlargement of the size of the sensors, which is a disadvantage. In its experimental study of T. L. Young [16], obtained from a supply voltage of $7 \mathrm{~V}$, that the variation of the offset voltage versus temperature for the diaphragm size is $800 \mu \mathrm{m}$ 
$\mathrm{X} 800 \mu \mathrm{m}$ and $20 \mu \mathrm{m}$ in thickness: it is $0.7 \mathrm{mV} /{ }^{\circ} \mathrm{C}$. In this case, the Joule heating provokes a temperature of $90{ }^{\circ} \mathrm{C}$. Therefore, the value of the offset voltage created by this effect is $121.9 \mathrm{mV}$, considering the offset of the bridge at $30{ }^{\circ} \mathrm{C}$ that was approximately $79.9 \mathrm{mV}[16]$.

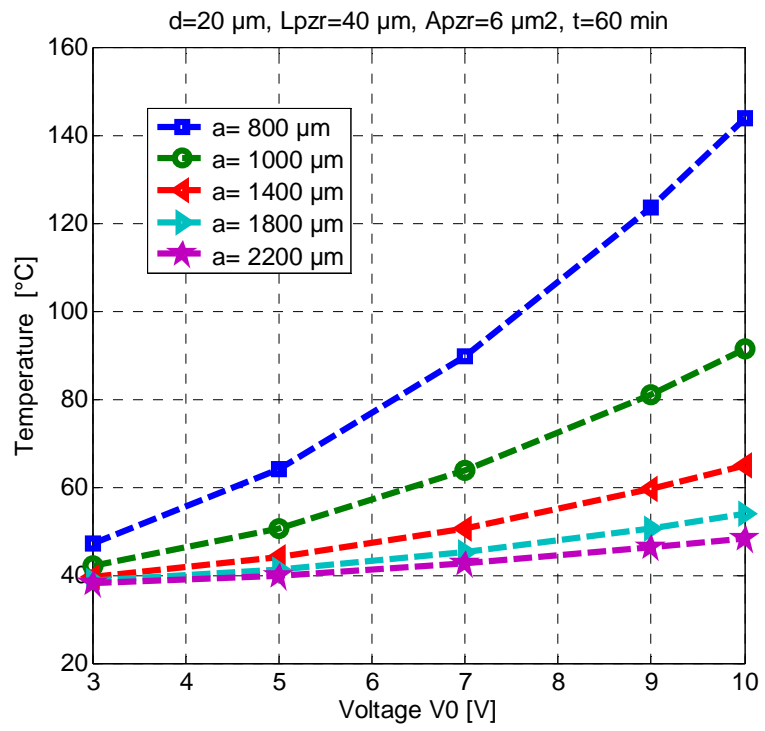

Figure 7. Temperature variation vs applied voltage for several side lengths of the diaphragm $a$

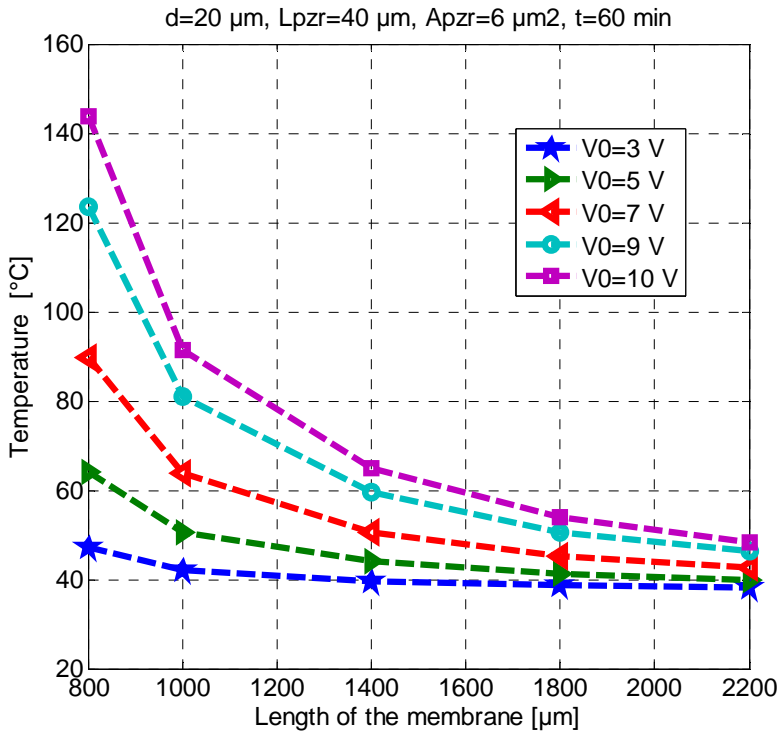

Figure 8. Variation temperature as a function of side length for different values of voltage $V_{0}$

\subsubsection{Effect of the operating time of the temperature rise in the sensor}

In order to acknowledge the evolution of the temperature created by joule heating for a period of 3 hours, we analyze the temperature rise by varying several geometrical parameters. As we can see from the below Figures, that after operating the device for a period ranging from 0 to $180 \mathrm{~min}$ by applying a voltage of $5 \mathrm{~V}$, the temperature takes a steady state value beyond 100 min and make it independent at the time. It has been observed in Figure 9 that the rise in temperature goes on decreasing as the length of the piezoresistor is increased. 


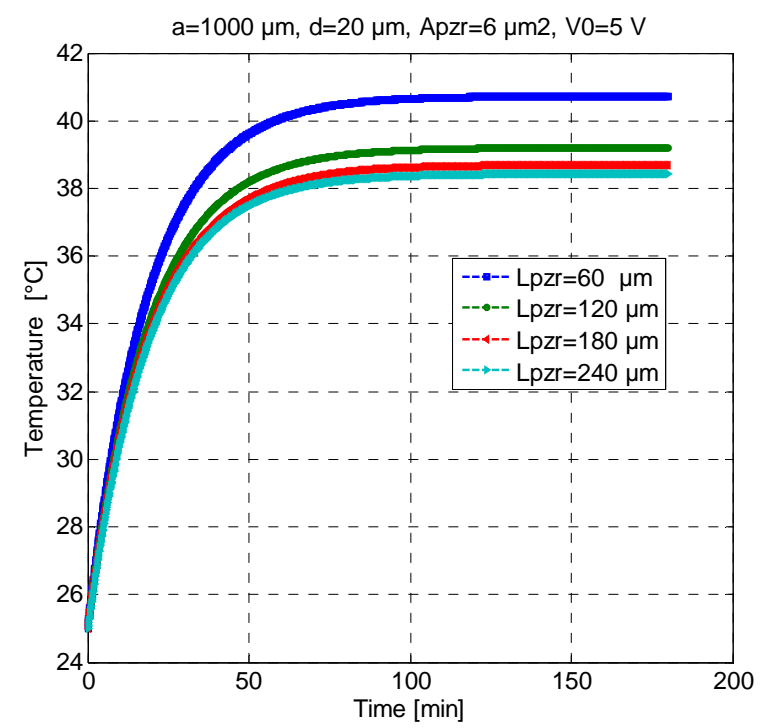

Figure 9. Temperature rise for various lengths of the piezoresistor $\boldsymbol{L}_{p z r}$

On the other hand, we can easily observe in Figures 10 and 11, respectively, that the temperature rise is decreasing function of the membrane side length and diaphragm thickness. According to these Figures, the two parameters have an enormous impact on the effect of Joule heating. So, when these parameters are great, this is leading to lessen the Joule heating. However, these parameters are themselves limited by other technological factors of manufacture such as: the dimensions of the device, the precision and reliability.

We may observe that these figures are analogous to the circuit constituting the loading of a capacitor connected to a voltage source through a resistor. In fact, this circuit may be exploited in the future works for obtaining the temperature rise due to Joule heating.

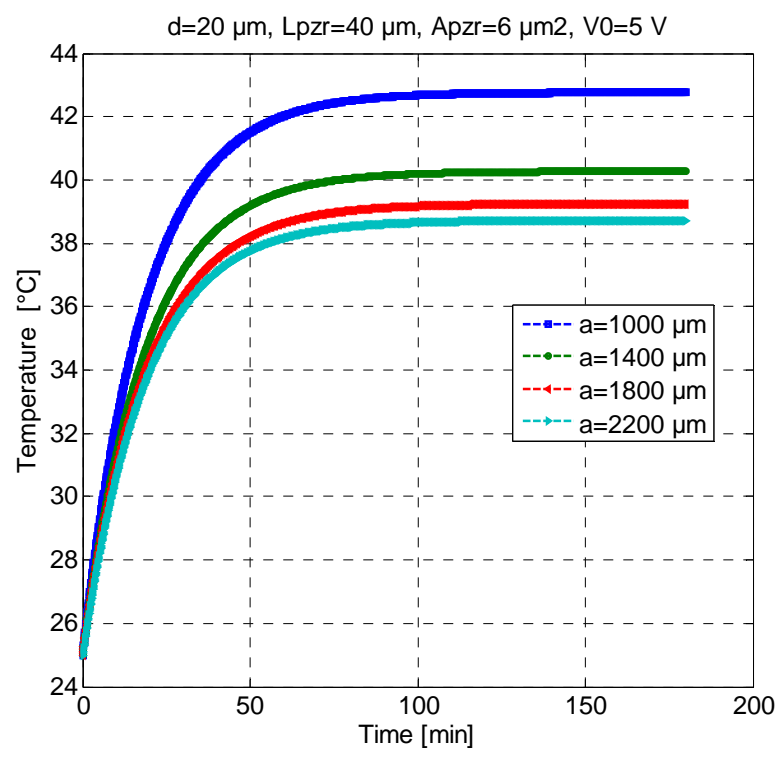

Figure 10. Temperature rise for different membrane side lengths 


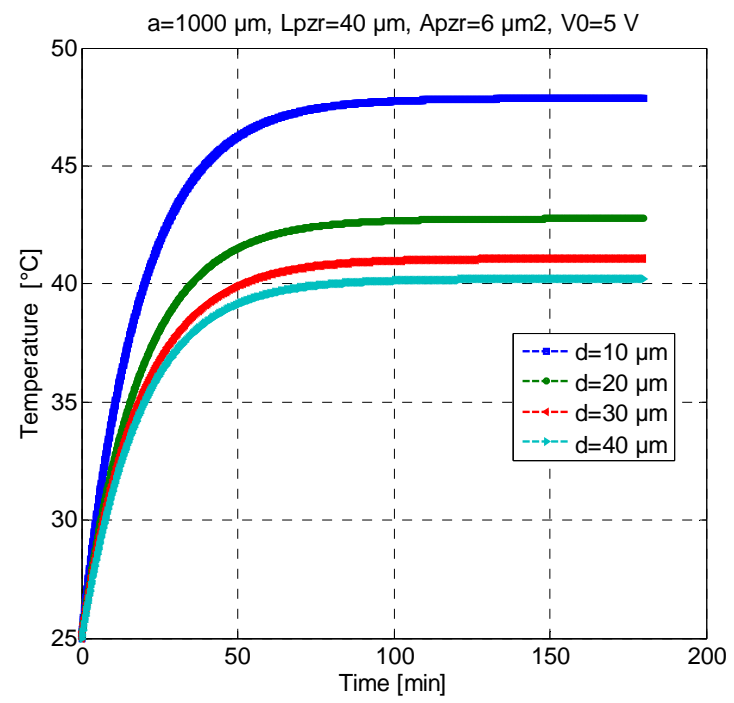

Figure 11. Temperature rise for several membrane thicknesses $d$

\section{CONCLUSION}

Provide the modeling of Joule heating in piezoresistive pressure sensors is essential to predict their thermal drift. To this end, the present study investigated the effect of applied voltage and the geometric parameters of temperature. In this paper we have developed a numerical model to reduce the Joule heating in such sensors types using Finite Difference Method (FDM). The model established gives an opportunity to study the temperature rise caused by Joule heating of sensors characteristics. We attempted to study the geometric influence parameters on these characteristics to optimize the sensor performance. The results confirmed that low bias voltage should be applied to reduce the Joule heating. Results showed also that the change in temperature is increasing function of the applied voltage. In addition, the Joule heating is inversely proportional to length of the piezoresistor and it's an increasing function of the cross-sectional area. So, a sensors with the low Joule heating, is obtained for a great length of the piezoresistor which is a drawback.

The Joule heating goes on decreasing as the membrane thickness is increased. But this affects greater the pressure sensitivity of the device. The temperature rise created by Joule heating in a sensor is a decreasing function of the side length of the membrane. Therefore, to minimize this effect, it is necessary to have a large side length. This option is easy to implement and does not affect the sensitivity to pressure. However, it gives large size defects, which is a drawback. On the other hand, to highlight the effect of the operating time of the device, various geometrical parameters have been used. The results showed that the Joule heating is reduced substantially for a short operating time. Besides, the temperature takes a steady state value beyond $100 \mathrm{~min}$ and will be independent of the time. Finally, this study allows us to optimize the sensor performance in function to the application for which it is dedicated.

\section{ACKNOWLEDGEMENTS}

The authors thank Dr. Merouani Lazher and Mr. Zahrouri Ahmed for their invaluable help for this project, which was fully done at the Electronics' Department, MoDERNa Laboratory, University of Constantine 1, Algeria. We also thank all the staff members of MoDERNa Laboratory.

\section{REFERENCES}

[1] D. Pittaya, et al., "Al Microheater and Ni Temperature Sensor Set based-on Photolithography with Closed-Loop Control," International Journal of Electrical and Computer Engineering (IJECE), vol. 5, pp. 849-858, 2015.

[2] Y. Huiyang, et al., "The intelligent compensation calibration algorithm for 3D polyhedron of the temperature drift of the silicon piezoresistive pressure sensor," 2015 IEEE International Conference on Electron Devices and SolidState Circuits (EDSSC), pp. 744 - 747, 2015.

[3] H. Jianqiu, "Design and Application of a High Sensitivity Piezoresistive Pressure Sensor for Low Pressure Conditions," Sensors, vol. 15, pp. 22692-22704, 2015.

[4] N. H. Duong, et al., "Advanced Liquid-Free, Piezoresistive, SOI-Based Pressure Sensors for Measurements in Harsh Environments," Sensors, vol.15, pp. 20305-20315, 2015. 
[5] S. Yicai, et al., "Electric drift of the bridge offset for pressure sensors and its utilization," Sensors and Actuators A, vol. 58, pp. 249-256, 1997.

[6] X. Dacheng, et al., "A temperature compensation algorithm of piezoresistive pressure sensor and software implementation," Mechatronics and Automation, IEEE ICMA 2013 Conference International Scientific Advisory Board, pp. 1738 - 1742, 2013.

[7] U. Aljancic, et al., "Temperature effects modeling in silicon piezoresistive pressure sensor," IEEE Melecon, Egypt, pp. 7-9, 2002.

[8] B. Abdelaziz, et al., "The Effect of Temperature and Doping Level on the Characteristics of Piezoresistive Pressure Sensor," Journal of Sensor Technology, vol. 4, pp. 59-65, 2014.

[9] M. Z. Ansari and C. Chongdu, "An Analytical Model of Joule Heating in Piezoresistive Microcantilevers," Sensors, vol. 10, pp. 9668-9686, 2010.

[10] M. Z. Ansari and C. Chongdu, "A conduction-convection model for self-heating in piezoresistive microcantilever biosensors," Sensors and Actuators A, vol. 175, pp.19-27, 2012.

[11] B. Abdelaziz, et al., "Thermo mechanical Modeling of Piezoresistive Pressure Sensor," International Review on Modelling and Simulations (I.RE.MO.S.), vol. 7, pp. 517-522, 2014.

[12] B. Abdelaziz, et al., "'hermal drift characteristics of capacitive pressure sensors," Journal of Engineering Science \& Technology, (in press).

[13] A. Arkan, et al., "Numerical Investigation of Heat Transfer Enhancement in a Circular Tube with Rectangular Opened Rings," Bulletin of Electrical Engineering and Informatics, vol. 4, pp. 18-25, 2015.

[14] S. Himanshu, et al., "Development and Simulation of Stand Alone Photovoltaic Model Using Matlab/Simulink," International Journal of Power Electronics and Drive System (IJPEDS), vol. 6, pp. 703-711, 2015.

[15] C. Pramanik, et al., "Development of SPICE Compatible Thermal Model of Silicon MEMS Piezoresistive Pressure Sensor for CMOS- MEMS Integration,” IEEE Sensors, Korea, pp. 22-25, 2006.

[16] L. Y. Tae, et al., "Compensation method of offset and its temperature drift in silicon piezoresistive pressure sensor using double wheatstone bridge configuration," The 8th International Conference on Solid-State Sensors and Actuators, and Eurosensors IX. Stockholm, Sweden, pp. 25- 29, 1995. 